urine) were $3.9 \mu \mathrm{g} / \mathrm{mg}$ and $7.5 \mu \mathrm{g} / \mathrm{mg}$ in men and women, respectively. The investigators demonstrated that participants with a UACR at or above the sex-specific median level were at a significantly higher risk of experiencing a cardiovascular event than those with lower levels of urinary albumin excretion (adjusted hazard ratio 2.92; 95\% Cl 1.57-5.44; $P<0.001$ ). A 1.75-fold increase in the mortality risk was also shown in the former group, although this difference did not reach statistical significance (95\% Cl 0.95-3.22; $P=0.08$ ).

Ärnlöv and colleagues comment that lowgrade urinary albumin excretion (below the threshold defining microalbuminuria) is an indicator of glomerular endothelial dysfunction and might predict cardiovascular disease. These findings suggest, therefore, that a reassessment of the current diagnostic threshold for microalbuminuria might be appropriate.

Ruth Kirby

Original article Ärnlöv J et al. (2005) Low-grade albuminuria and incidence of cardiovascular disease events in nonhypertensive and nondiabetic individuals: the Framingham Heart Study. Circulation 112: 969-975

\section{Delays to carotid endarterectomy in a UK population}

It is now well established that carotid endarterectomy is of most benefit when performed promptly after a stroke or transient ischemic attack. As soon as 2 weeks after the event, the risk of recurrent stroke rises sharply. Nevertheless, studies have shown that delays to endarterectomy are common. This point is underlined in a recent study, which also questions the reasons for such delays and assesses the consequences.

The first part of the analysis included 853 patients (from a total population of approximately 690,000 in Oxfordshire, UK), who underwent carotid imaging following a retinal or cerebral transient ischemic attack or stroke. The median interval between the presenting event and referral for carotid imaging was 9 days, and a median interval of 33 days was noted between the event and imaging actually taking place. Importantly, a median of 100 days elapsed between presentation and endarterectomy. Next, the investigators recorded the incidence of recurrent stroke prior to surgery in 38 patients with $\geq 50 \%$ symptomatic carotid stenosis. This analysis revealed an increase in the risk of recurrent stroke from $21 \%$ at 2 weeks after the event, to $32 \%$ at 12 weeks.

Authors Fairhead et al. note that these delays to carotid imaging and endarterectomy are similar to those seen elsewhere, and are mostly accounted for by secondary care. They urge that surgery, if appropriate, should be carried out within 1 week of the presenting event, to minimize the risk of recurrent stroke.

Ruth Kirby

Original article Fairhead JF et al. (2005) Population-based study of delays in carotid imaging and surgery and the risk of recurrent stroke. Neurology 65:371-375

\section{Longer duration of anticoagulation reduces the risk of recurrence of venous thromboembolism}

The optimal duration of anticoagulation for patients with venous thromboembolism (VTE) remains controversial. Previous studies, and even meta-analyses, have come to conflicting conclusions on the risks and benefits of prolonging anticoagulation in these patients.

A literature search of online databases enabled Ost et al. to identify 15 randomized, controlled trials that assessed recurrence of VTE in patients receiving anticoagulation therapy of different durations. Studies involving only highrisk patients were excluded. In addition, four trials were excluded from the primary analysis as they did not include follow-up of patients after anticoagulation therapy was discontinued; however, these patients were included in the analysis of the risk of recurrence with lifelong compared with fixed-duration therapy.

A random-effects model showed that the risk of recurrence of VTE was lower in patient groups continuing lifelong anticoagulation than in those receiving fixed-duration therapy (0.106 fewer VTE events per person-year; $P<0.001)$. The risk of major bleeding was similar in the two groups. Interestingly, the benefit of having prolonged anticoagulation was maintained even after therapy was discontinued, although the reduction in the VTE event rate was not as pronounced as that achieved with ongoing therapy $(0.020$ fewer VTE events per person-year; $P=0.04$ ). 